Background Since the emergence of COVID-19, the academic and scientific community has reacted at pace to understand its epidemiology, diagnosis, treatment, prevention and impact. There are concerns that in the panic to get answers to help manage the pandemic, many of the cornerstones of robust methods are being omitted. The aim of this review was to map the nature, scope and quality of evidence syntheses on COVID-19 and to explore the relationship between review quality and the extent of researcher, policy and media interest.

Methods We conducted a systematic review of systematic reviews, rapid reviews, overviews and qualitative evidence syntheses addressing a research question relating to COVID-19. Searches were conducted in PubMed, Epistemonikos COVID19 evidence, the Cochrane Library of Systematic Reviews, The Cochrane COVID-19 Study Register, EMBASE, CINAHL, Web of Science Core Collection, and the WHO COVID-19 database in June 2020. Abstract and full text screening were undertaken by two independent reviewers. Descriptive information on review type, purpose, population, size, citation and attention metrics were extracted along with whether the review met six key methodological criteria. For reviews meeting all six methodological criteria, additional data were extracted on methods and publication metrics and AMSTAR-2 was used to assess the quality of the reported methods. Registration: PROSPERO CRD42020188822

Results Searches returned 2334 unique records. After applying eligibility criteria we included 280 reviews. Less than half reported undertaking critical appraisal and a third had no reproducible search strategy. There was considerable overlap in topics, with discordant findings. Eighty-eight of the 280 reviews met all six methodological criteria. Of these, 3 were rated as of moderate or high quality on AMSTAR-2, with the majority having critical flaws: only a third reported registering a protocol, and less than one in five searched named COVID19 databases. Review conduct and publication was rapid, with 56 of the 88 systematic review reported as being conducted within three weeks, and half published within three weeks of submission. Despite being of low quality and many lacking robust methods, the reviews received substantial attention across both academic and public platforms, and the attention was not related to the quality of review methods.

Conclusion Methodological flaws limit the validity of systematic reviews and the generalisability of their findings. Yet by being reported as 'systematic reviews', many readers may well regard them as high quality evidence, irrespective of the methods undertaken. To maintain trustworthiness, researchers, peerreviewers and journal editors need to ensure systematic reviews adhere to guidelines of best practice.

\section{OP32 JOB LOSS AND LOWER HEALTHCARE UTILIZATION DUE TO COVID-19 AMONG OLDER ADULTS ACROSS 27 EUROPEAN COUNTRIES}

${ }^{1}$ Gabriela Ksinan Jiskrova*, 1,2 Martin Bobák, 1,2Hynek Pikhart, 'Albert J Ksinan. ${ }^{1}$ RECETOX Faculty of Science, Masaryk University, Brno, Czech Republic; ${ }^{2}$ Department of Epidemiology and Public Health, University College London, London, UK

\subsection{6/jech-2021-SSMabstracts.32}

Background Older adults are one of the population groups at the highest risk of severe illness from COVID-19. However, little is known about the impact of the pandemic on economic activity and healthcare utilization for reasons unrelated to
COVID-19 among older adults. This study aimed to examine the prevalence and predictors of unemployment due to COVID-19 and healthcare utilization during the pandemic in a sample of older adults across 27 European countries. We examined the associations between individual and household demographic characteristics as well as country-level characteristics and the likelihood of the outcomes.

Methods We utilized cross-sectional data from the large multinational Study of Health, Ageing and Retirement in Europe (SHARE) COVID-19 Survey, collected between June and August 2020. All participants $(n=52,061)$ reported whether they forwent medical treatment and whether their appointment was postponed due to COVID-19. Economically active participants $(\mathrm{n}=10,958)$ reported whether they lost a job due to COVID-19. Three-level hierarchical models were estimated for each outcome to test the effects of individual, household, and country-level characteristics.

Results The mean prevalence of reported job loss, forgone, and postponed medical care were 19\%, 12\%, and 26\%, respectively. Women we more likely to lose their job than men (OR 1.27; 95\% CI 1.14-1.41 at mean age) and this effect was larger for older women. Covid-related job loss was also associated with household income (OR per 1,000 EUR was 0.84 ; 95\% CI $0.78-0.90$ ) and lower education (OR comparing primary vs. tertiary education was 1.27 ; 95\% CI 1.14 1.41). Forgone and postponed medical care was associated with older age in men, female sex, and higher education. For example, women were more likely to forgo medical treatment compared to men (OR 1.63; 95\% CI 1.54-1.73 at mean age). At the country level, postponed medical care was associated with more stringent governmental anti-COVID measures (OR for inter-quartile range of stringency index was $1.48 ; 95 \% \mathrm{CI}$ 1.14-1.93).

Conclusion Job loss and lower healthcare utilization for nonCOVID-19 related reasons were prevalent among older adults in the SHARE sample and were associated with several sociodemographic and country characteristics. Job loss appeared to disproportionally affect already economically vulnerable individuals, which may contribute to an exacerbation of social inequalities over time. Additionally, the results highlighted the importance of focusing on maintaining access to healthcare during the lockdown and following up on any missed medical appointments to prevent increased morbidity due to missed screenings and treatment.

\section{OP33 DIGITAL EXCLUSION DURING THE COVID-19 PANDEMIC IN THE ENGLISH LONGITUDINAL STUDY FOR AGEING POPULATION}

Shivan Thakrar*, Jennifer Cole, Helen Parretti, Nicholas Steel. Public Health and Health Services Research, University of East Anglia, Norwich, Norfolk, UK

\subsection{6/jech-2021-SSMabstracts.33}

Background The NHS long term plan commits to 'digital first primary care' by 2024. Increasing reliance on digital access may disadvantage those who do not use the internet. We aimed to assess changes in internet use in adults over 50 years of age before and during the coronavirus pandemic.

Methods Participants in the English Longitudinal Study for Ageing were asked how often they used the internet or email in Wave 9 (W9) from June 2018 to June 2019 and COVID Wave 1 (CW1) from June to July 2020. Response options 